White Paper for the Planetary Decadal Survey 2023-2032

\title{
Electrostatic Dust Transport Effects on Shaping the Surface Properties of the Moon and Airless Bodies across the Solar System
}

July 15,2020

\author{
Lead Author: \\ $\mathrm{Xu}$ Wang \\ University of Colorado - Boulder \\ Phone: 303-217-3699 \\ Email: xu.wang@colorado.edu

\section{Co-Authors:} \\ David T. Blewett, JHU-APL \\ Georgiana Kramer, Planetary Science Institute \\ Donald Barker, NASA JSC and Jacobs Technology Inc. \\ Christine Hartzell, University of Maryland \\ Daoru Han, Missouri University of Science and Technology \\ Mihaly Horányi, University of Colorado - Boulder \\ Ian Garrick-Bethell, University of California, Santa Cruz \\ Hsing-Wen Hsu, University of Colorado - Boulder \\ Joseph Wang, University of Southern California \\ Rhushik Chandrachud, Mumbai University \\ Devanshu Jha, MVJ College of Engineering \\ Adrienne Dove, University of Central Florida \\ Amara Graps, Planetary Science Institute
}




\section{Summary}

Electrostatic dust charging and subsequent transport on the lunar surface due to direct exposure to the solar wind plasma and solar radiation is a more than five-decade-old problem. Dust activity has been suggested as an explanation for several lunar surface observations from the beginning in the Apollo era. Beyond the Moon, related observations indicate that this electrostatic process may be a universal phenomenon on airless bodies across the solar system. Recent studies have made important breakthroughs in understanding the fundamentals of dust charging, mobilization and lofting mechanisms, offering strong support for its occurrence on the surfaces of airless bodies. This white paper reviews historical and recent observational evidence, reports recent findings, examines knowledge gaps and outstanding science questions, and provides recommendations for future studies. Special attention is given to in situ measurements over the next decade in order to ultimately solve the outstanding questions and, more importantly, understand the primary implications for the evolution of the surfaces of the Moon and other airless bodies across the solar system. Furthermore, potential risks posed by electrostatic dust transport to future surface exploration of these bodies, especially any long-term human presence on the lunar surface, needs to be well characterized in order to define and implement appropriate risk mitigation strategies and methods through proper systems design and testing.

\section{Scientific Motivation, Current Understanding and Outstanding Questions}

Airless bodies, including the Moon, asteroids, comets and moons of other planets, generally have no/a tenuous atmosphere and no global magnetic field. Many of these bodies are covered by a layer of fractured rock and mineral particles, called regolith. The regolith is directly exposed to the space environment and undergoes various space weathering processes such as micrometeoroid bombardment, thermal fatigue, solar wind implantation and sputtering, and cosmic radiation. In addition, dust particles in the regolith are charged by the solar wind plasma and photoemission due to solar radiation. Hence, dust may be mobilized, lofted and transported due to electrostatic forces. A number of observations on the Moon and other airless bodies have indicated the occurrence of this electrostatic process, which has attracted increased attention because this process may play a significant role in shaping the physical and spectral properties of the regolith on these bodies.

\subsection{Lunar Observations Related to Electrostatic Dust Transport}

The first evidence of electrostatic dust transport was the Lunar Horizon Glow (LHG), which was inferred from observations recorded by the TV cameras onboard several Surveyor lunar landers in late 1960s, for example from Surveyor 7 in Fig. 1 (left). The bright glow about $30 \mathrm{~cm}$ above the lunar surface was believed to be due to sunlight forward scattered by a cloud of dust particles $(\sim 10 \mu \mathrm{m}$ in diameter) that were electrostatically levitated above the surface in the lunar terminator region [Criswell, 1973]. This lofting height is shown to be consistent with a recent observation by the Chang'e-3 rover, which identifies a layer of fine dust on lunar rocks up to 28 cm high [Yan et al., 2019].

Later, evidence for such electrostatic dust activity was obtained by instrument measurements and astronaut observations taken during the Apollo missions. The Lunar Ejecta and Meteorites Experiment (LEAM), deployed on the lunar surface, registered what has been interpreted as up to hundreds of hits per day of low-speed $(<100 \mathrm{~m} / \mathrm{s})$ dust particles, mostly during terminator crossings, as opposed to a few hits per day of hypervelocity $(1-25 \mathrm{~km} / \mathrm{s})$ cosmic dust particles [Berg et al., 1976]. However, later work analyzing different LEAM datasets [Grün and Horányi, 2013], found no significant rate enhancement associated with the terminator crossing. 

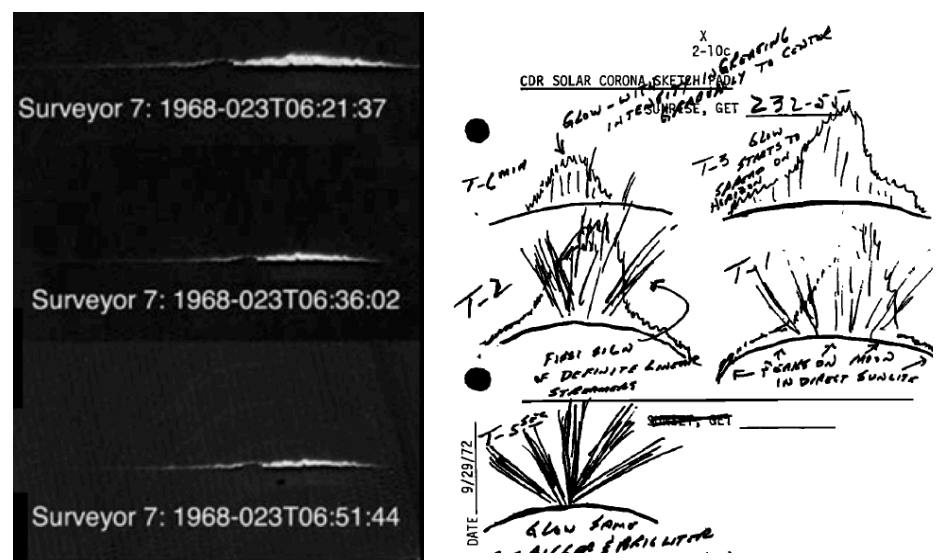

Fig. 1. Left: Lunar Horizon Glow images from National Space Science Data Center; Right: Sketches by the Apollo 17 astronaut E. A. Cernan showing a large-scale horizon glow (appears as a shoulder on the corona and zodiacal light in the central bulge) and high-altitude streamers [ Zook and McCoy, 1991].

A large-scale horizon glow and high-altitude streamers illustrated in sketches by Apollo astronauts (Fig. 1, right) were suggested to be attributed to submicron sized $(\sim 0.1 \mu \mathrm{m}$ in radius) dust particles lofted between 10-100 $\mathrm{km}$ altitude through electrostatic mechanisms [McCoy and Criswell, 1974; Zook and McCoy, 1991]. Though the Apollo remote-sensing observations also indicate an excessive brightness in the zodiacal light [McCoy, 1976; Glenar et al., 2011], such a dense dust population was neither indicated from the remote sensing observations by Clementine [Glenar et al., 2014] and LRO/LAMP [Feldman et al., 2014], nor indicated from the in-situ measurements by LADEE/LDEX

[Szalay and Horányi, 2015]. Contrarily, LADEE/UVS intermittently observed a dense cloud of nanometer-sized particles originating from the lunar surface [Wooden et al., 2016].

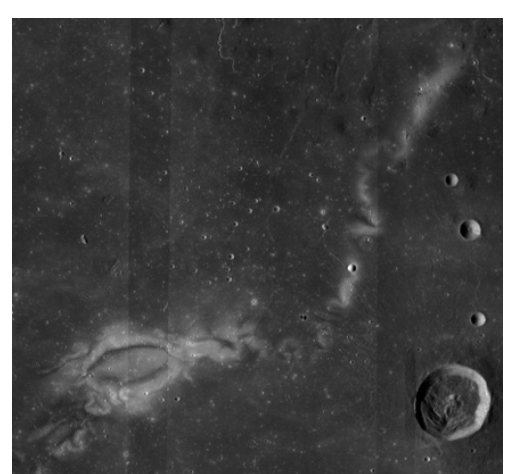

Fig. 2. LRO image of the Reiner Gamma Swirl.

Swirl-shaped, high-albedo markings on the lunar surface (Fig. 2), the so-called lunar swirls, have recently attracted great attention because they represent locations with especially high science values in several aspects [Blewett et al., White Paper, 2020; Kramer et al., White Paper, 2020]. Electrostatic dust transport is one of the hypotheses for the origin of these swirls. It has been suggested that electrostatically lofted dust particles may be sorted by electric fields created as a result of solar wind plasma interactions with lunar magnetic anomalies at the location of the swirls, forming high-albedo patterns due to the increased relative brightness of fine dust particles [GarrickBethell et al., 2011].

\subsection{Dust Transport Phenomena on Airless Bodies across the Solar System}

Beyond the Moon, a number of observations on other airless bodies also indicate the occurrence of electrostatic dust transport and mobilization. Dust ponds observed on asteroid 433 Eros (Fig. 3, left [Robinson et al., 2001]) and Comet 67P [Thomas et al., 2015] were suggested to be caused by the deposition of electrostatically transported dust into craters on these bodies [Colwell et al., 2005]. Intermittently appearing 'spokes' in Saturn's rings (Fig. 3, center [Smith et al., 1981]) were explained as a result of dust particles electrostatically lofted above the ring plane as a footprint of dust impact generated plasma spreading radially [Morfill et al., 1983]. Dust mobilization was hypothesized to be responsible for the highly smooth surface on Saturn's icy moon Atlas (Fig. 3, right [Hirata and Miyamoto, 2012]) and the high surface-porosity indicated from thermal emissivity features of main-belt asteroids and Jupiter Trojans [Vernazza et al., 2012]. 
Recent Hayabusa2 observations show the absence of submillimeter-sized dust on asteroid Ryugu [Jaumann et al., 2019], possibly due to electrostatic dust escape. Lack of fine-grained regolith was also observed on asteroids Itokawa by Hayabusa [Yano et al., 2006] and Bennu by OSIRIS-REx [Lauretta et al., 2019].
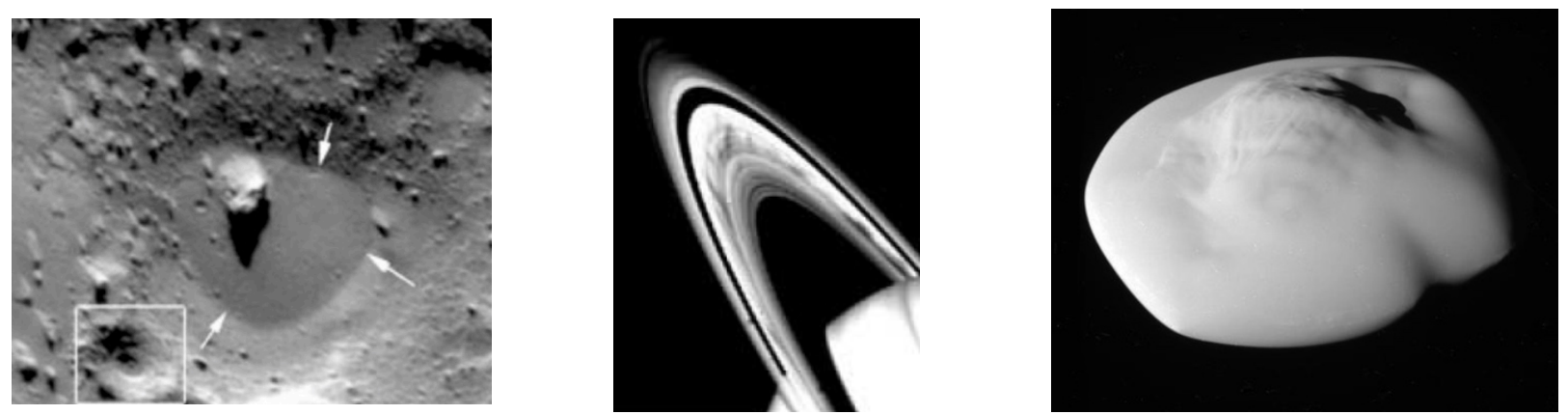

Fig. 3. Left: A dust pond (pointed by three arrows) on asteroid 433 Eros [Robinson et al., 2001]; Center: Radial 'spokes' in Saturn's rings [Smith et al., 1981]; and Right: Highly smooth surface on Saturn's icy moon Atlas [Hirata and Miyamoto, 2012].

\subsection{Current Understanding}

Motivated by these observations of natural phenomena, electrostatic dust charging and lofting has been explored through laboratory experiments, theoretical studies, and computer simulations. This includes modeling studies of the dynamics of charged dust in response to various electrical environments at various airless bodies. Charging of dust particles on surfaces in various plasma conditions was investigated through both laboratory experiments and by theoretical efforts, and dust mobilization and transport were demonstrated in these laboratory experiments. However, until recently, most models of dust lofting dynamics were based on assumptions of initial lofting conditions such as initial charge and launch velocity. While observed in the laboratory, the fundamental problem of how dust particles obtain sufficiently large charge to initially become lofted or mobilized on airless bodies, remained a challenge to theoretical explanation.
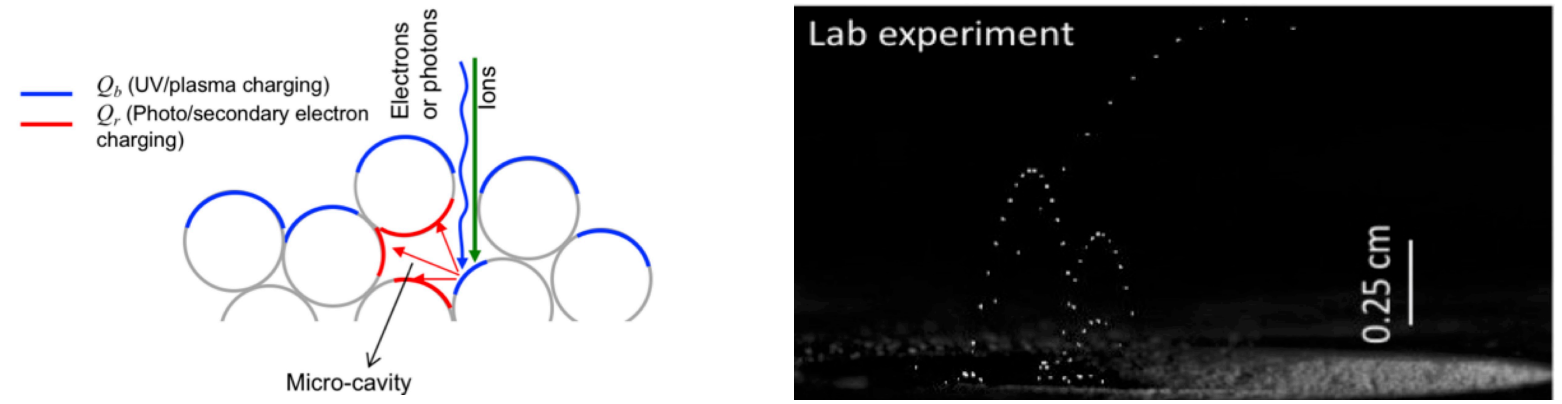

Fig. 4. Left: Patched Charge Model for regolith dust particles [Wang et al., 2016]; and Right: Trajectories of electrostatically lofted dust particles [Wang et al., 2016].

Recent laboratory experiments and associated theoretical work have revolutionized our understanding of dust lofting. A new Patched Charge Model (Fig. 4, left) developed based on advanced laboratory experiments (Fig. 4, right) [Wang et al., 2016] has shifted our view from the charging process on the regolith surface to microstructures within the regolith. It was shown that the emission and re-absorption of photoelectrons and/or secondary electrons inside microcavities between dust particles can result in a buildup of large, negative charges on the surrounding 
particles, such that the subsequent strong repulsive forces between these particles cause their lofting or mobilization. Computer simulations [Zimmerman et al., 2016] have since also demonstrated charging enhancement due to strong grain-scale electric fields in the regolith. Since then, additional laboratory experiments have produced results in support of the Patched Charge Model [Schwan et al., 2017; Hood et al., 2018; Dove et al., 2018; Orger et al., 2019].

These laboratory experiments have provided new insights into the characteristics of charging and lofting of regolith dust, including the initial charge and launch velocity, the lofting rate, and the size distribution. It is shown that the charging and lofting process largely depends on ambient plasma conditions, as well as the regolith and dust properties, including regolith compaction and microstructure, dust size, shape and composition, and inter-particle cohesion [Hartzell and Scheeres, 2011; Hartzell et al., 2013; Wang et al., 2016].

\subsection{Outstanding Science Questions}

Recent laboratory studies have demonstrated results indicating strong support for the occurrence of electrostatic dust transport on the surfaces of the Moon and other airless bodies. Though these results have greatly advanced our knowledge about this phenomenon, laboratory experiments cannot fully recreate conditions on the surfaces of these bodies. Additionally, as shown from the laboratory results, the charging and lofting process can be largely affected by the unique and varying regolith and dust properties, as well as the ambient plasma environment. Dust transport and mobilization is therefore expected to show unique behaviors on different types of bodies (e.g., the Moon, asteroids, and comets). The first outstanding question is:

- What are the dynamics of dust charging and subsequent lofting and transport in the near-surface plasma and electric field environment on different types of airless bodies?

The answer to the first question will provide critical inputs in investigating the role of electrostatic dust transport in shaping the surface properties of airless bodies. As indicated from existing observations and recent laboratory studies, this electrostatic process may efficiently alter surface morphology and porosity, lead to dust size sorting, and drive dust migration. This phenomenon is more pronounced on small bodies because of their low gravity, which can cause global-scale dust transport and even escape. Subsequently, both surface thermal properties (e.g., thermal emissivity and thermal inertia) and spectral properties that are sensitive to the upper most layer of regolith [Delbo et al., 2015; Pieters et al., 1993] are expected to be significantly altered due to these changes in the regolith physical properties. The second outstanding question is:

- How does electrostatic dust transport play a role in shaping the surface physical and spectral properties on different types of airless bodies?

\section{Significance}

2.1. Surface Evolution of the Moon and Airless Bodies across the Solar System

As described above, the importance of electrostatic dust transport on resurfacing processes of airless bodies has attracted increased attention since the recent breakthroughs in understanding the fundamental mechanisms of this phenomenon and the accumulation of observations from recent missions. These electrostatic processes potentially add a new dimension, in addition to impacts and space weathering, for understanding the surface evolution of the Moon and other airless bodies.

Studies of this topic address a top science question in the previous 2012-2023 Planetary Science Decadal Survey: How have the myriad chemical and physical processes that shaped the solar system operated, interacted, and evolved over time? and the SMD Planetary Science Goal in 
the NASA Science Plan 2014: Explore and observe the objects in the solar system to understand how they formed and evolve. Specifically, these studies address a science goal in Lunar SCEM 2007 and LEAG's ASM-SAT reports: Science Goal 8b - Determine the size, charge, and spatial distribution of electrostatically transported dust grains and assess their likely effects on lunar exploration and lunar-based astronomy; and Transformative Lunar Science 2018: 2f) Evaluate the extended record of space weather and fundamental processes of plasma interactions with surfaces.

\subsection{Dust hazards to future surface exploration}

Mobilized dust due to natural mechanisms and/or human activity pose potential risks to future surface exploration, including operations, and hardware and crew safety. As reported from the Apollo missions, dust particles can readily stick to all surfaces, such as spacesuits, optical and thermal systems and components. A number of problems during the Apollo missions have been attributed to dust loading [Afshar-Mohajer et al., 2015], including damage to spacesuits due to the abrasiveness of lunar dust, degradation of radiators, issues with thermal control systems and retroreflectors, and interference with hatch seals and Extravehicular Activity (EVA) systems. In addition, dust brought back to living quarters could lead to serious health risks if inhaled by astronauts over long durations. Dust hazards have been recognized by the Lunar Surface Innovation Consortium (LSIC) as one of the major technical challenges for future human and robotic exploration on the lunar surface, especially for long-term human presence. Electrostatic dust mobilization and lofting is also of concern to asteroid space resource utilization [Graps, et al. 2016; Graps et al., 2019]. Behavior of charged dust needs to be well characterized in order to define appropriate operations, design, testing, and mitigation strategies and methods.

Studies in this topic address 1) Science Goal 8b in Lunar SCEM 2007 and LEAG's ASMSAT reports, as described above; 2) HEOMD Lunar SKG-III-D-2: Regolith adhesion to human systems and associated mechanical degradation; 3) Strategic Objective 2.2 in the NASA 2018 Strategic Plan: Conduct human exploration in Deep Space, including to the surface of the Moon; and 4) Global Exploration Roadmap: Enable sustained living/working around and on the Moon.

\subsection{Planetary defense for Near-Earth-Objects (NEOs)}

As described above, fine dust particles significantly contribute to the regolith thermal properties, which plays a role in determining orbital and rotational dynamics of small bodies. Studies in this topic are potentially applicable to NASA's NEO survey programs for planetary defense. These studies are within the scope of the NASA's 2018 Strategic Objective 1.1: Understand The Sun, Earth, Solar System, And Universe - Safeguarding and Improving Life on Earth; and NASA's Explore Science 2020-2024 Priority 1 Exploration and Scientific Discovery, Strategy 1.1 - Planetary Science: Advances in planetary science, coupled with leading efforts to detect, track, and characterize near-Earth objects, will continue to improve planetary defense.

\section{Recommendations}

3.1. Synergy of laboratory experiments, theoretical modeling and in situ measurements

To answer the outstanding science questions described in Section 1.4, in situ measurements will play a leading role in future studies to 1) unambiguously confirm the occurrence of the electrostatic dust lofting phenomenon on airless bodies; and 2) quantitatively characterize the dust charging and lofting processes on different types of airless bodies. It is recommended to support payloads on future lunar landed missions as well as asteroid and/or comet missions to characterize this electrostatic dust phenomenon. Due to the complexity of the problem, in situ measurements 
need to be adequately supported by laboratory experiments and theoretical modeling. More importantly, models of electrostatic dust transport are needed to study its effects on the evolution of the surface physical and spectral properties of airless bodies. These studies will help explain existing observation mysteries and guide future missions that include space resource utilization and/or study regolith properties, especially sample return missions. It is recommended to support laboratory experiments and computational models that complement in situ measurements.

\subsection{Lunar landed missions}

Future lunar landed missions (crewed and robotic), including both Commercial Lunar Payload Services (CLPS) and large-lander missions as part of the Artemis program, provide accessibility to perform in situ measurements on the lunar surface. These missions are expected to provide firstof-its-kind measurements to determine the ground truth of electrostatic dust transport, which will be the next milestone towards answering this long-standing problem. Dedicated in situ dust instruments combined with plasma instruments are recommended to be deployed to a variety of locations on the lunar surface to characterize and compare dust activities in different regolith and plasma conditions. Additionally, remote sensing measurements are recommended to investigate dust at different altitudes over a global scale. A combination of these investigations will paint a comprehensive picture about lunar dust transport.

\subsection{Asteroid and/or comet missions with small landers/rovers}

As described above, due to low gravity, dust lofting on small bodies like asteroids and comets is expected to manifest larger effects on altering their surface properties than the Moon. Dust lofting is also expected to be largely different between asteroids and comets due to their different regolith properties. It is recommended to perform in situ investigations on both types of bodies using small landers or rovers carrying dedicated dust instruments deployed by large asteroid or comet missions. Examples of this type of mission implementation include Rosetta, Hayabusa2 and upcoming Martian Moons eXploration (MMX) missions. As smallsat technologies develop, standalone smallsat missions (e.g., SIMPLEx) are also recommended to be implemented to measure electrostatic dust transport on NEOs.

\subsection{Dust mitigation}

As described above, dust hazards to future surface exploration and operations on the Moon and asteroids need to be well characterized through laboratory and modeling studies as well as in situ investigations. Characterizations of charged dust mobilized by natural mechanisms and/or human activities will help develop the deployment and operation strategies, design of any payloads and hardware that interact with the lunar or asteroid surface, and appropriate dust removal methods, especially methods based on electrodynamics. Collaborations between SMD and HEOMD are recommended to tackle this challenge.

\section{References}

Blewett, D. T., et al. (2020), The Science Case for Lander or Rover Missions to a Lunar Magnetic Anomaly/Swirl, Decadal Survey White Paper 2023-2032.

Kramer, G., et al. (2020), The Plethora of Science Afforded by a Lunar Swirl, Decadal Survey White Paper 2023-2032.

Afshar-Mohajer, N., C.-Y. Wu, J. S. Curtis, J. R. Gaier (2015), Adv. Space Res., 56, 1222-1241. 
Berg, O. E., H. Wolf, and J. Rhee (1976), Interplanetary Dust and Zodiacal Light, Lect. Notes Phys., vol. 48, edited by H. Elsaesser and H. Fechtig, pp. 233-237, Springer, Berlin. Colwell, J. E., Gulbis, A. A. S., Horányi, M., Robertson, S. (2005), Icarus 175, 159-169.

Criswell, D. R. (1973), Photon and Particle Interactions with Surfaces in Space, edited by R. J. L. Grard, pp. 545-556, Springer, New York.

Delbo, M., Mueller, M., Emery, J.P., Rozitis, B., Capria, M. T. (2015), Asteroids IV, edited by

Michel, P., DeMeo, F. E., Bottke, W. F., pp. 107-128, University of Arizona Press.

Dove, A., M. Horányi, S. Robertson, X. Wang (2018), Planet. Space Sci., 156, 92-95.

Feldman, P. D., et al. (2014), Icarus, 233, 106-113.

Garrick-Bethell, I., J. W. Head III, and C. M. Pieters (2011), Icarus, 212, 480-492.

Glenar, D. A., T.J. Stubbs, J.E. McCoy, R.R. Vondrak (2011), Planet. Space Sci., 59, 1695-1707.

Glenar, D. A., T. J. Stubbs, M. Hahn, Y. Wang (2014), J. Geophys. Res., 119, 2548-2567.

Graps, A.L., et al. (2019). ASIME 2018 White Paper: In-Space Utilisation of Asteroids:

"Asteroid Composition: Answers to Questions from the Asteroid Miners."

https://arxiv.org/abs/1904.11831

Graps, A.L., et al. (2016). ASIME 2016 White Paper: In-Space Utilisation of Asteroids:

"Answers to Questions from the Asteroid Miners" https://arxiv.org/abs/1612.00709

Grün, E., and M. Horányi (2013), Planet. Space Sci., 89, 2-14.

Hartzell, C. M., and D. J. Scheeres (2011), Planet. Space Sci., 59, 1758-1768.

Hartzell, C. M., X. Wang, D.J. Scheeres, M. Horányi (2013), Geophys. Res. Lett., 40, 1038-1042

Hirata, N., and H. Miyamoto (2012), Icarus, 220, 106-113.

Hood, N., et al. (2018), Geophys. Res. Lett., 45, 13,206-13,212.

Jaumann, R., et al. (2019), Science, 365, 817-820.

Lauretta, D.S., et al. (2019), Nature, 568, 55-60.

McCoy, J. E., and D. R. Criswell (1974), 5th Lunar Sci. Conf., 1571, 2991-3005, Houston, TX.

McCoy, J. E. (1976), Proceedings of 7th Lunar and Planetary Science Conference, Houston,

Tex., vol. 1, edited by R. B. Merrill, pp. 1087-1112, Pergamon Press, New York.

Morfill, G. E., E. Grün, C.K. Goertz, and T.V. Johnson (1983), Icarus, 53, 230-235.

Orger, N. C., Toyoda, K., Masui, H., Cho, M. G., (2019), Adv. Space Res., 63, 3270-3288.

Pieters, C. M., E. M. Fischer, O. Rode and A. Basu (1993), J. Geophys. Res., 98, 20,817-20,824.

Robinson, M. S., P. C. Thomas, J. Veverka, S. Murchie, B. Carcich (2001), Nature, 413, 396-400

Schwan, J., X. Wang, H.-W. Hsu, E. Grün, and M. Horányi (2017), Geophys. Res. Lett., 44, 3059-3065.

Smith, B. A., et al. (1981), Science, 212, 163-191.

Szalay, J. R., and M. Horanyi (2015), Geophys. Res. Lett., 42, 5141-5146.

Thomas, N., et al. (2015), Astron. Astrophys., 583, A17.

Vernazza, P., et al. (2012), Icarus, 221, 1162-1172.

Wang, X., J. Schwan, H.-W. Hsu, E. Grün, and M. Horányi (2016), Geophys. Res. Lett., 43, 6103-6110.

Wooden, D., Cook, A. M., Colaprete, A., Glenar, D. A., Stubbs, T. J., Shirley, M., (2016), Nature Geoscience, 9, 665-668.

Yan, Q., et al. (2019), Geophys. Res. Lett., 46, 9405-9413.

Yano, H., et al. (2006), Science, 312, 1350-1353.

Zimmerman, M. I., Farrell, W. M., Hartzell, C. M., Wang, X., Horanyi, M., Hurley, D. M., Hibbitts, K. (2016), J. Geophys. Res., 121, 2150-2165.

Zook, H. A., and J. E. McCoy (1991), Geophys. Res. Lett., 18, 2117-2120. 